\title{
NNMSM type-II and -III
}

\author{
Naoyuki Haba ${ }^{1,2}$, Kunio Kaneta ${ }^{2,3,4}$, Ryo Takahashi ${ }^{2, a}$ \\ ${ }^{1}$ Graduate School of Science and Engineering, Shimane University, Matsue, Shimane 690-8504, Japan \\ 2 Department of Physics, Faculty of Science, Hokkaido University, Sapporo, Hokkaido 060-0810, Japan \\ ${ }^{3}$ Kavli Institute for the Physics and Mathematics of the Universe (WPI), University of Tokyo, Kashiwa, Chiba 277-8568, Japan \\ ${ }^{4}$ Department of Physics, Graduate School of Science, Osaka University, Toyonaka, Osaka 560-0043, Japan
}

Received: 30 September 2013 / Accepted: 6 December 2013 / Published online: 24 January 2014

(C) The Author(s) 2014. This article is published with open access at Springerlink.com

\begin{abstract}
We suggest two types of extension of the standard model, which are the so-called next to new minimal standard model type-II and -III. They can achieve gauge coupling unification as well as suitable dark matter abundance, small neutrino masses, baryon asymmetry of the universe, inflation, and dark energy. The gauge coupling unification can be realized by introducing two or three extra new fields, and they could explain charge quantization. We also show that there are regions in which the vacuum stability, coupling perturbativity, and correct dark matter abundance can be realized with current experimental data at the same time.
\end{abstract}

\section{Introduction}

The discovery of the Higgs particle at the Large Hadron Collider (LHC) experiment $[1,2]$ filled the last piece of the standard model (SM). Furthermore, the results from the LHC experiment are almost consistent with the SM, and no signatures of the supersymmetry (SUSY) have been discovered. However, there are some unsolved problems in the SM, e.g., the SM does not have a dark matter (DM) candidate although a SUSY model can give it. The SUSY is one of the excellent candidates beyond the SM, because it can also solve the gauge hierarchy problem in addition to the DM. Moreover, gauge coupling unification (GCU) can be realized in a minimal supersymmetric standard model (MSSM). But the discovery of the Higgs boson with a $126 \mathrm{GeV}$ mass and no signatures of the SUSY may disfavor the SUSY at low energy.

Actually, various extensions of the SM without the SUSY have been proposed. One minimal extension is the new minimal standard model (NMSM) [3]. The NMSM contains a gauge singlet real scalar, two right-handed neutrinos, an inflaton, and a small cosmological constant, which can explain

\footnotetext{
a e-mail: ryo.takahasi88@gmail.com
}

the DM, small neutrino masses, and baryon asymmetry of the universe (BAU), inflation, and dark energy (DE), respectively. ${ }^{1}$ The next to new minimal SM (NNMSM) has been suggested in Ref. [10]. The NNMSM adds new particle contents to the NMSM to explain the gauge coupling unification (GCU). These are two adjoint fermions and four vector-like fermions. ${ }^{2}$ In the NNMSM, the stability and triviality conditions have also been analyzed by use of recent experimental data of Higgs and top masses [11]. As a result, it could be found that there are parameter regions in which the correct abundance of DM can also be realized at the same time. One is the lighter DM mass, region: $63.5 \mathrm{GeV} \lesssim m_{S} \lesssim 64.0 \mathrm{GeV}$, and the other is the heavier one: $708 \mathrm{GeV} \lesssim m_{S} \lesssim 2040 \mathrm{GeV}$ (writing $m_{S}$ for the lighter DM mass) with the center value of top pole mass.

Reference [10] has introduced six new fields, which were assumed to be at the same mass scale, to realize GCU. In this work, we also extend the NMSM and leave the condition of the same mass scale for the new fields adopted in the NNMSM. As a result, we can reduce the particle contents of the NNMSM while realizing GCU and vacuum stability, and satisfying phenomenological constraints such as $\mathrm{DM}$, small neutrino masses, BAU, inflation, $\mathrm{DE}$, and the proton decay. We suggest two types of model, the first model includes two adjoint fermions for the GCU and the second one has three adjoint fermions. The degrees of freedom of additional fermions in the models decrease compared to the NNMSM. We call the models NNMSM type-II (two adjoint fermions) and NNMSM type-III (three adjoint fermions), respectively. The NNMSM type-II also includes two righthanded neutrinos like the NNMSM, and the type-III does not have them. Both models have a gauge singlet scalar,

\footnotetext{
${ }^{1}$ See also [4-9] for other extensions of the SM.

2 The model did not address the gauge hierarchy problem because the magnitude of the fine-tuning is much smaller than that of the cosmological constant problem.
} 
inflaton, and a small cosmological constant. We also analyze the stability and triviality bounds with the $126 \mathrm{GeV}$ Higgs mass, the recent updated limits on the DM particle [12], and the latest experimental value of the top pole mass, $173.5 \mathrm{GeV}$ in both models. We will point out that there are parameter regions in both models where the stability and triviality bounds, the correct abundance of DM, and the Higgs and top masses can be realized at the same time.

This paper is organized as follows: In Sect. 2, we suggest the NNMSM type-II and show that the model can realize GCU. Then, the vacuum stability and DM are investigated. Some related arguments in the model such as the inflation, neutrino, and baryogenesis are also given. In Sect. 3, we suggest the NNMSM type-III. In particular, we focus on two simple models in this type of model. Similar phenomenological arguments to those in type-II are also presented. Section 4 is devoted to the summary. We give other setups for the realization of GCU in the Appendix.

\section{NNMSM type-II}

\subsection{Model}

We suggest a next to new minimal standard model typeII (NNMSM-II) by reducing the particle contents of the NNMSM [10], which has two adjoint fermions $\lambda_{a}(a=2,3)$, four vector-like fermions $L_{i}^{\prime}\left(\overline{L_{i}^{\prime}}\right)(i=1,2)$, the gauge singlet real scalar boson $S$, two right-handed neutrinos $N_{i}$, the inflaton $\varphi$, and a small cosmological constant $\Lambda$ in addition to the SM. Our model removes four vector-like fermions from the NNMSM, but adds a new energy scale to the model. The quantum numbers of these particles are given in Table 1. Only the gauge singlet scalar particle has odd parity under an additional $Z_{2}$ symmetry, while the other additional particles have even parity. We will show that the singlet scalar becomes DM as in the NNMSM. The runnings of the gauge couplings are changed from the SM due to new particles with charges. The realization of GCU is one of the important results of this work as we will show later.

We consider NNMSM-II as a renormalizable theory, and thus the most general form of the Lagrangian allowed by the symmetries and renormalizability is given by

Table 1 Quantum numbers of the additional particles in NNMSM-II $(i=1,2)$

\begin{tabular}{llllll}
\hline & $\lambda_{3}$ & $\lambda_{2}$ & $S$ & $N_{i}$ & $\varphi$ \\
\hline$S U(3)_{C}$ & 8 & 1 & 1 & 1 & 1 \\
$S U(2)_{L}$ & 1 & 3 & 1 & 1 & 1 \\
$Z_{2}$ & + & + & - & + & + \\
\hline
\end{tabular}

$$
\mathcal{L}_{\mathrm{NNMSM}}=\mathcal{L}_{\mathrm{SM}}+\mathcal{L}_{S}+\mathcal{L}_{N}+\mathcal{L}_{\varphi}+\mathcal{L}_{\Lambda}+\mathcal{L}^{\prime}
$$

$\mathcal{L}_{\mathrm{SM}} \supset-\lambda\left(|H|^{2}-\frac{v^{2}}{2}\right)^{2}$,

$\mathcal{L}_{S}=-\frac{\bar{m}_{S}^{2}}{2} S^{2}-\frac{k}{2}|H|^{2} S^{2}-\frac{\lambda S}{4 !} S^{4}$ + (kinetic term),

$\mathcal{L}_{N}=-\left(\frac{M_{R i} i}{2} \overline{N_{i}^{c}} N_{i}+h_{v}^{i \alpha} \overline{N_{i}} L_{\alpha} \tilde{H}+\right.$ c.c. $)$ $+($ kinetic term)

$$
\begin{aligned}
\mathcal{L}_{\varphi}= & -B \varphi^{4}\left[\ln \left(\frac{\varphi^{2}}{\sigma^{2}}\right)-\frac{1}{2}\right]-\frac{B \sigma^{4}}{2}-\mu_{1} \varphi|H|^{2} \\
& -\mu_{2} \varphi S^{2}-\kappa_{H} \varphi^{2}|H|^{2}-\kappa_{S} \varphi^{2} S^{2} \\
& -\left(y_{N}^{i j} \varphi \overline{N_{i}} N_{j}+y_{3} \varphi \overline{\lambda_{3}} \lambda_{3}+y_{2} \varphi \overline{\lambda_{2}} \lambda_{2}+\text { c.c. }\right) \\
& +(\text { kinetic term }), \\
\mathcal{L}_{\Lambda}= & \left(2.3 \times 10^{-3} \mathrm{eV}\right)^{4}, \\
\mathcal{L}^{\prime}= & \left(y_{v_{\alpha}} \overline{L_{\alpha}} \lambda_{2} \tilde{H}+M_{3} \overline{\lambda_{3}} \lambda_{3}+M_{2} \overline{\lambda_{2}} \lambda_{2}+\text { h.c. }\right) \\
& +(\text { kinetic terms }),
\end{aligned}
$$

with $\alpha=e, \mu, \tau$ and $\tilde{H}=i \sigma_{2} H^{*}$ where $\mathcal{L}_{\mathrm{SM}}$ is the Lagrangian of the SM, which includes the Higgs potential. $v$ is the vacuum expectation value (VEV) of the Higgs boson: $v=246 \mathrm{GeV}$. $\mathcal{L}_{S, N, \varphi, \Lambda}$ are Lagrangians for the $\mathrm{DM}$, righthanded neutrinos, inflaton, and the cosmological constant, respectively. $\mathcal{L}_{\mathrm{SM}}+\mathcal{L}_{S, N, \Lambda}$ are the same as those of the NNMSM. ${ }^{3} \mathcal{L}^{\prime}$ is a new Lagrangian in NNMSM-II where $L_{\alpha}$ is for left-handed lepton doublets in the SM. ${ }^{4}$

\subsection{Gauge coupling unification}

First, we investigate the runnings of the gauge couplings in the NNMSM-II. Since we introduce two adjoint fermions, $\lambda_{3}$ and $\lambda_{2}$, listed in Table 1, the beta functions of the RGEs for the gauge couplings become

$2 \pi \frac{\mathrm{d} \alpha_{j}^{-1}}{\mathrm{~d} t}=b_{j}$

where $t \equiv \ln (\mu / 1 \mathrm{GeV}), \mu$ is the renormalization scale, and $\alpha_{j} \equiv g_{j}^{2} /(4 \pi)(j=1,2,3)$ with $g_{1} \equiv \sqrt{5 / 3} g^{\prime}$. The beta

\footnotetext{
${ }^{3}$ For the present cosmic acceleration, we simply assume that the origin of DE is a tiny cosmological constant, which is given in $\mathcal{L}_{\Lambda}$ of Eq. (6), so that NNMSM-II predicts the equation of state parameter as $\omega=-1$, like the NNMSM. We will not focus on the DE in this work anymore.

${ }^{4}$ If one assigns odd parity under $Z_{2}$ to $\lambda_{2}$ and does not introduce $S$, only the neutral component of $\lambda_{2}$ can be a DM candidate. The field content in the scenario without $S$ decreases compared to the NNMSM-II but it becomes difficult to satisfy the vacuum stability and triviality bounds with the center values of the Higgs and top masses at the same time. Since the $S$ also plays a crucial role for the discussions of the stability and triviality bounds in addition to the DM candidate as shown later, we focus on the model with $S$.
} 


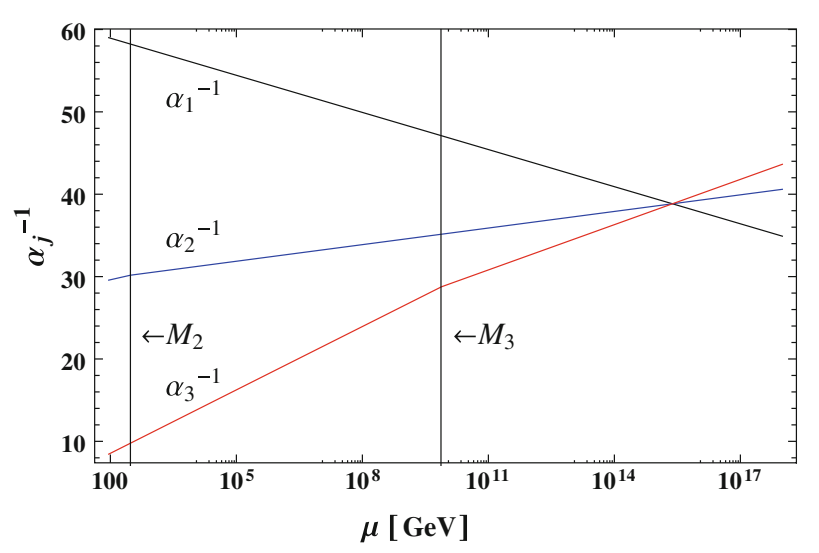

Fig. 1 The runnings of the gauge couplings in NNMSM-II. The horizontal axis is the renormalization scale and the vertical axis is for the values of $\alpha_{i}^{-1}$. The runnings of $\alpha_{1}^{-1}, \alpha_{2}^{-2}$, and $\alpha_{3}^{-1}$ are described by black, blue, and red solid curves, respectively. We take $M_{3} \simeq 7.44 \times 10^{9} \mathrm{GeV}$ and $M_{2}=300 \mathrm{GeV}$, and the coupling unification is realized at $\Lambda_{\mathrm{GCU}} \simeq 2.41 \times 10^{15} \mathrm{GeV} \mathrm{GeV}$ with $\alpha_{\mathrm{GCU}}^{-1} \simeq 38.8$

functions from the SM and the new particles contribute to $b_{j}$. Each contribution from the SM and the new particles shown in Table 1 is given by

$$
\begin{aligned}
\left(b_{1}^{\mathrm{SM}}, b_{2}^{\mathrm{SM}}, b_{3}^{\mathrm{SM}}\right) & =\left(\frac{41}{10},-\frac{19}{6},-7\right), \\
\left(b_{1}^{\lambda_{3}}, b_{2}^{\lambda_{3}}, b_{3}^{\lambda_{3}}\right) & =(0,0,2), \\
\left(b_{1}^{\lambda_{2}}, b_{2}^{\lambda_{2}}, b_{3}^{\lambda_{2}}\right) & =\left(0, \frac{4}{3}, 0\right) .
\end{aligned}
$$

The NNMSM has assumed the same mass scale for the new particles, $M_{\mathrm{NP}}=M_{3}=M_{2}=M_{L_{i}^{\prime}}$, where $M_{L_{i}^{\prime}}$ are the masses of the vector-like fermions. On the other hand, we allow different mass scales for the two adjoint fermions in NNMSM-II.

According to the numerical analyses, taking for the two masses $M_{3} \simeq 7.44 \times 10^{9} \mathrm{GeV}$ and $M_{2}=300 \mathrm{GeV}$ can realize GCU with a good precision at 1-loop level as shown in Fig. 1. ${ }^{5}$

The beta functions in NNMSM-II are

$$
b_{j}= \begin{cases}b_{j}^{\mathrm{SM}} & \text { for } M_{Z} \leq \mu<M_{2} \\ b_{j}^{\mathrm{SM}}+b_{j}^{\lambda_{2}} & \text { for } M_{2} \leq \mu<M_{3} . \\ b_{j}^{\mathrm{SM}}+b_{j}^{\lambda_{2}}+b_{j}^{\lambda_{3}} & \text { for } M_{3} \leq \mu\end{cases}
$$

We show the thresholds of new particles with $M_{3} \simeq 7.44 \times$ $10^{9} \mathrm{GeV}$ and $M_{2}=300 \mathrm{GeV}$ by black solid lines. The realization of GCU by adding these adjoint fermions was pointed

\footnotetext{
5 In this analysis, we take the following values [11]: $\sin ^{2} \theta_{\mathrm{W}}\left(M_{Z}\right)=$ $0.231, \alpha_{\mathrm{em}}^{-1}\left(M_{Z}\right)=128$, and $\alpha_{\mathrm{S}}\left(M_{Z}\right)=0.118$, for the parameters in the EW theory, where $\theta_{\mathrm{W}}$ is the Weinberg angle, $\alpha_{\mathrm{em}}$ is the fine structure constant, and $\alpha_{\mathrm{s}}$ is the strong coupling, respectively.
}

out in Ref. [4]. The NNMSM-II suggests the GCU at

$\Lambda_{\mathrm{GCU}} \simeq 2.41 \times 10^{15} \mathrm{GeV}$,

with the unified coupling

$\alpha^{-1} \simeq 38.8$.

A constraint from the proton decay experiments is $\tau$ ( $p \rightarrow$ $\left.\pi^{0} e^{+}\right)>8.2 \times 10^{33}$ years [11]. When we suppose the minimal $S U$ (5) GUT at $\Lambda_{\mathrm{GCU}}$, the protons decay of $p \rightarrow \pi^{0} e^{+}$ occurs by exchanging heavy gauge bosons of the GUT gauge group. The partial decay width of the proton for $p \rightarrow \pi^{0} e^{+}$ is estimated as

$$
\begin{aligned}
\Gamma\left(p \rightarrow \pi^{0} e^{+}\right)= & \alpha_{\mathrm{H}}^{2} \frac{m_{\mathrm{p}}}{64 \pi f_{\pi}^{2}}(1+D+F)^{2}\left(\frac{4 \pi \alpha_{\mathrm{GCU}}}{\Lambda_{\mathrm{GCU}}} A_{\mathrm{R}}\right)^{2} \\
& \times\left(1+\left(1+\left|V_{u d}\right|^{2}\right)^{2}\right),
\end{aligned}
$$

where $\alpha_{\mathrm{H}}^{2}$ is the hadronic matrix element, $m_{\mathrm{p}}$ is the proton mass, $f_{\pi}$ is the pion decay constant, $D$ and $F$ are the chiral Lagrangian parameters, $A_{\mathrm{R}}$ is the renormalization factor, and $V_{u d}$ is an element of the CKM matrix (e.g., see $[4,13,14])$. In our analysis, we take these parameters as $m_{\mathrm{p}}=0.94 \mathrm{GeV}, f_{\pi}=0.13 \mathrm{GeV}, A_{\mathrm{R}} \simeq 1.02$, $D=0.80$, and $F=0.47$. The theoretical uncertainty on the proton lifetime comes from the hadron matrix elements, $\alpha_{\mathrm{H}}=-0.0112 \pm 0.0034 \mathrm{GeV}^{3}[15]$. When $\alpha_{\mathrm{H}}$ is taken as a smaller value, which is $\alpha_{\mathrm{H}}=-0.0146 \mathrm{GeV}^{3}$, the proton lifetime is predicted as small, and this case gives a conservative limit. At the point determined by (11) and (12), the proton lifetime can be evaluated as $\tau \simeq 5.19 \times 10^{33}\left(1.82 \times 10^{34}\right)$ years for $\alpha_{\mathrm{H}}=-0.0146(-0.0078) \mathrm{GeV}^{3}$. Thus, the value of $\alpha_{\mathrm{H}}=-0.0078 \mathrm{GeV}^{3}$ can satisfy the experimental bound from the proton decay although the conservative case $\left(\alpha_{\mathrm{H}}=-0.0146 \mathrm{GeV}^{3}\right)$ cannot. For the center value of $\alpha_{\mathrm{H}}=-0.0112 \mathrm{GeV}^{3}$, the proton lifetime is evaluated as $\tau \simeq 8.55 \times 10^{33}$ years, which also satisfies the experimental limit. Since the future Hyper-Kamiokande experiment is expected to exceed the lifetime $\mathcal{O}\left(10^{35}\right)$ years [16], which corresponds to $\Lambda_{\mathrm{GCU}} \simeq 4.42_{-0.73}^{+0.63} \times 10^{15} \mathrm{GeV}$ for $\alpha_{\mathrm{H}}=-0.0112 \pm 0.0034 \mathrm{GeV}^{3}$, proton decay is observed if NNMSM-II is correct.

When we take a larger value of $M_{2}$, such as $800 \mathrm{GeV}, \mathrm{GCU}$ can be realized with a larger value of $M_{3}, 2.03 \times 10^{10} \mathrm{GeV}$. However, GCU with a larger value of $M_{2}$ leads to a smaller scale of $\Lambda_{\mathrm{GCU}}$, and thus the constraint from the proton decay becomes stronger. In fact, GCU with $M_{2}=800 \mathrm{GeV}$ leads to a proton lifetime of $\tau \simeq(2.14-7.48) \times 10^{33}$ years for $\alpha_{\mathrm{H}}=$ $-0.0112 \pm 0.0034 \mathrm{GeV}^{3}$, which is ruled out by the proton decay experiment. Therefore, the upper bounds on the adjoint fermions masses are $\left(M_{3}, M_{2}\right) \lesssim\left(2 \times 10^{10}, 800\right) \mathrm{GeV}$. On the other hand, the LHC experiment gives a lower limit to the $S U(2)_{L}$ triplet mass $M_{2}$ of $245 \mathrm{GeV} \leq M_{2}$ by the ATLAS examining the channel of four lepton final states 
(and $(180-210) \mathrm{GeV} \leq M_{2}$ by the CMS for the three lepton final states) $[17,18]$. As a result, the allowed region for $M_{2}$ in NNMSM-II is $245 \mathrm{GeV} \leq M_{2} \lesssim 800 \mathrm{GeV}$. The corresponding region for $M_{3}$ is $7 \times 10^{9} \mathrm{GeV} \lesssim M_{3} \lesssim 2 \times 10^{10}$ $\mathrm{GeV}$ to realize GCU. Since the $S U(2)_{L}$ triplet fermion can be discovered up to $M_{2} \leq 750 \mathrm{GeV}$ by the LHC with $\sqrt{s}=14$ $\mathrm{TeV}$ [19], most of the mass region of $M_{2}$ in the NNMSM-II can be checked by the experiment.

\subsection{Abundance and stability of new fermions}

Next, we discuss the abundance and stability of new fermions, $\lambda_{3}$ and $\lambda_{2} . \lambda_{3}$ is expected to be long lived since it cannot decay into the SM sector. A stable colored particle is severely constrained by experiments with heavy isotopes, since it is bounded in nuclei and appears as anomalously heavy isotopes (e.g., see [20]). The number of the stable colored particles per nucleon should be smaller than $10^{-28}\left(10^{-20}\right)$ for its mass up to $1(10) \mathrm{TeV}[21,22]$. But the calculation of the relic abundance of the stable colored particle is uncertain because of the dependence on the mechanism of hadronization and nuclear binding [23].

In this paper, we apply the same scenario to avoid the problem of the presence of the stable colored particle as in the NNMSM. It is to consider few production scenario for the stable particle, i.e., if the stable particle were rarely produced in the thermal history of the universe and clear the constraints of the colored particle. In fact, a particle with a mass of $M$ is very rarely produced thermally if the reheating temperature after the inflation is lower than $M /(35 \sim 40) .{ }^{6}$ Therefore, we consider a reheating temperature $T_{\mathrm{RH}}$ of

$T_{\mathrm{RH}} \lesssim \frac{M_{3}}{40}=1.86 \times 10^{8} \mathrm{GeV}$

since $M_{3}=7.44 \times 10^{9} \mathrm{GeV}$. On the other hand, $\lambda_{2}$ is thermally produced but it can decay into the SM particles through the Yukawa interaction in Eq. (7) before the Big Bang nucleosynthesis $(\mathrm{BBN})$. The condition for the decay before the BBN is $\mathcal{O}\left(10^{-13}\right) \lesssim\left|y_{v_{\alpha}}\right|$, which can also be consistent with a constraint from neutrino mass generation as we will discuss later. Therefore, the presence of two new adjoint fermions in NNMSM-II for GCU is not problematic.

\subsection{Stability, triviality, and dark matter}

In this section, we investigate the parameter region where not only stability and triviality bounds but also correct abundance of the DM are achieved. The ingredients of Higgs and DM sector in NNMSM-II are the same as the NNMSM [10], which are given by $\mathcal{L}_{\mathrm{SM}}$ and $\mathcal{L}_{S}$ in Eqs. (2) and (3) but the

\footnotetext{
${ }^{6}$ We thank S. Matsumoto for pointing out this point in a private discussion.
}

runnings of the gauge couplings are different from those of the NNMSM. The singlet scalar $S$ becomes the DM also in NNMSM-II. Reference [10] has pointed out that there are two typical regions in which the vacuum stability, the correct abundance of DM, the $126 \mathrm{GeV}$ Higgs mass, and the latest experimental value of the top mass can be realized at the same time. One is the lighter mass region of DM and the other is the heavier one. It has also been shown that the top mass dependence is quite strong even within the experimental error of the top pole mass, $M_{\mathrm{t}}=173.5 \pm 1.4 \mathrm{GeV}$. The future XENON100 experiment with 20 times higher sensitivity will be able to rule out the lighter mass region completely. On the other hand, the heavier mass region can currently be allowed by all experiments searching for DM. The region will be ruled out or checked by the future direct experiments of XENON $100 \times 20$, XENON1T and/or combined data from indirect detections of Fermi+CTA+Planck at $1 \sigma \mathrm{CL}$.

In NNMSM-II, the runnings of gauge couplings are slightly changed with those of the NNMSM. Therefore, we reanalyze the vacuum stability, triviality, and the correct abundance of DM in NNMSM-II. The RGEs for three quartic couplings of the scalars [3] and the DM mass are given by

$$
\begin{aligned}
(4 \pi)^{2} \frac{\mathrm{d} \lambda}{\mathrm{d} t}= & 24 \lambda^{2}+12 \lambda y^{2}-6 y^{4}-3 \lambda\left(g^{\prime 2}+3 g^{2}\right) \\
& +\frac{3}{8}\left[2 g^{4}+\left(g^{\prime 2}+g^{2}\right)^{2}\right]+\frac{k^{2}}{2} \\
(4 \pi)^{2} \frac{\mathrm{d} k}{\mathrm{~d} t}= & k\left[4 k+12 \lambda+\lambda_{S}+6 y^{2}-\frac{3}{2}\left(g^{\prime 2}+3 g^{2}\right)\right] \\
(4 \pi)^{2} \frac{\mathrm{d} \lambda_{S}}{\mathrm{~d} t}= & 3 \lambda_{S}^{2}+12 k^{2}
\end{aligned}
$$

and

$m_{S}=\sqrt{\bar{m}_{S}^{2}+k v^{2} / 2}$

respectively. We should also use the present limits for the singlet DM model. We comment on Eq. (16): the right-hand side of the equation is proportional to $k$ itself. Thus, if we take a small value of $k\left(M_{Z}\right)$, the evolution of $k$ tends to be slow and remaining at a small value, and the running of $\lambda$ is close to that of the SM. In our analysis, the boundary conditions of the Higgs self-coupling and top Yukawa coupling are given by

$\lambda\left(M_{Z}\right)=\frac{m_{h}^{2}}{2 v^{2}}=0.131, \quad y\left(M_{t}\right)=\frac{\sqrt{2} m_{t}\left(M_{t}\right)}{v}$

for the RGEs, where the VEV of the Higgs field is $v=$ $246 \mathrm{GeV}$.

Let us solve the RGEs, Eqs. (15)-(17), and obtain the stable solutions, i.e., the scalar quartic couplings are within the range of $0<\left(\lambda, k, \lambda_{S}\right)<4 \pi$ up to the Planck scale 


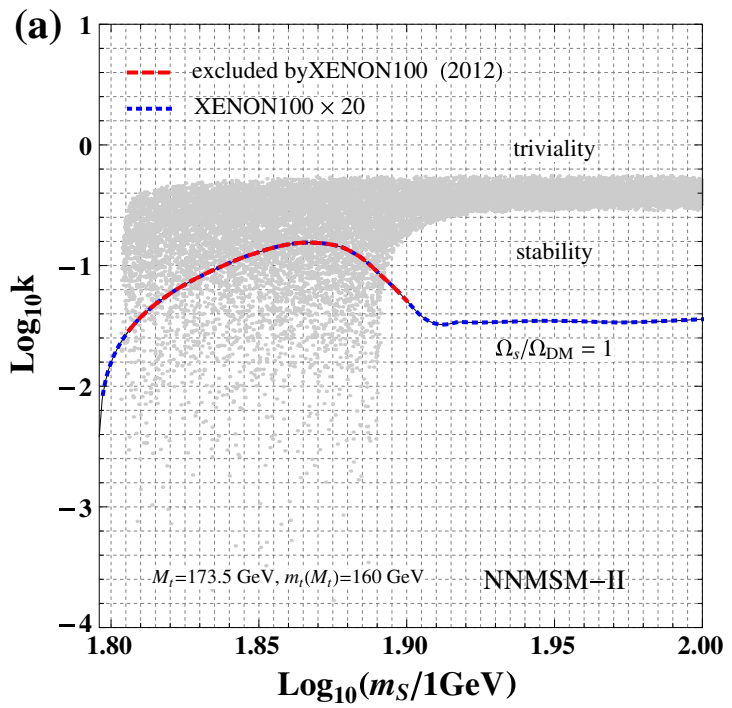

Fig. 2 A contour of fixed relic density $\Omega_{S} / \Omega_{\mathrm{DM}}=1$ and a region, which is described by gray plots, satisfying the stability and triviality bounds with $M_{t}=173.5 \mathrm{GeV}\left(m_{t}\left(M_{t}\right)=160 \mathrm{GeV}\right)$ in NNMSM-II. The boundaries of the plotted region are determined by the stability and triviality conditions. The words 'stability' and 'triviality' in both figures

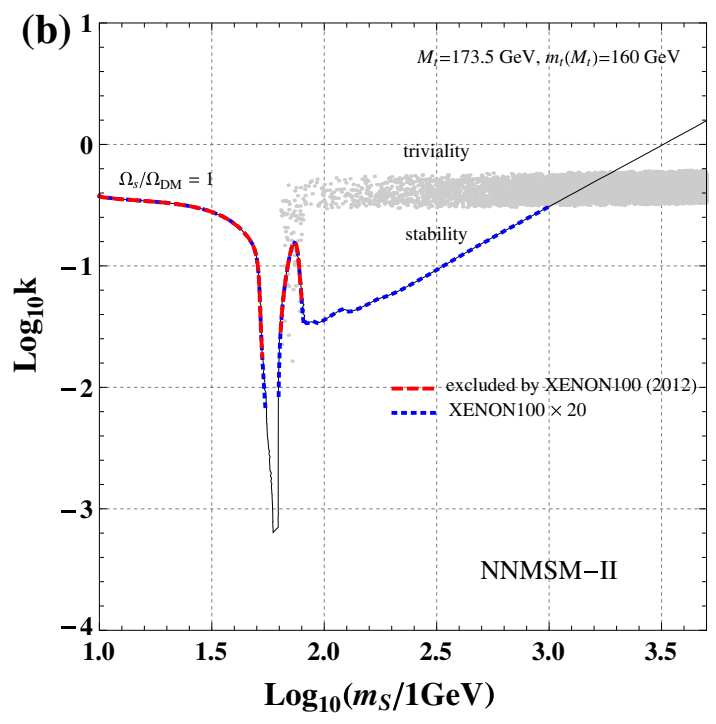

refer to the corresponding conditions. The (red) dashed, and (blue) dotted lines are experimental limits from XENON100 (2012) and 20 times higher sensitivity of XENON100, respectively. a The mass region is $63 \mathrm{GeV} \leq m_{S} \leq 100 \mathrm{GeV}\left(1.8 \leq \log \left(m_{S} / 1 \mathrm{GeV}\right) \leq 2.0\right)$. b The mass region is $10 \mathrm{GeV} \leq m_{S} \leq 5,000 \mathrm{GeV}\left(1.0 \leq \log \left(m_{S} / 1 \mathrm{GeV}\right) \leq 3.7\right)$
$M_{\mathrm{pl}}=10^{18} \mathrm{GeV}$. Figure 2 shows the case of a central value of $M_{t}=173.5 \mathrm{GeV}$ (leading to $m_{t}\left(M_{t}\right)=160 \mathrm{GeV}$ ).

The solutions of the RGEs are described by gray plots in Fig. 2, where the horizontal and vertical axes are sion limit [12], which is described by the (red) dashed line in Fig. $2 .^{7}$ There are two regions, $R_{1,2}$, which satisfy both the correct DM abundance and the triviality bound simultaneously,

$$
\begin{aligned}
R_{1} & = \begin{cases}63.5 \mathrm{GeV} \lesssim m_{S} \lesssim 64.0 \mathrm{GeV} & \left(1.803 \lesssim \log _{10}\left(m_{S} / 1 \mathrm{GeV}\right) \lesssim 1.806\right) \\
2.40 \times 10^{-2} \lesssim k\left(M_{Z}\right) \lesssim 2.63 \times 10^{-2} \quad\left(-1.64 \lesssim \log _{10} k\left(M_{Z}\right) \lesssim-1.58\right)\end{cases} \\
R_{2} & = \begin{cases}955 \mathrm{GeV} \lesssim m_{S} \lesssim 2040 \mathrm{GeV} & \left(2.98 \lesssim \log _{10}\left(m_{S} / 1 \mathrm{GeV}\right) \lesssim 3.31\right) \\
0.316 \lesssim k\left(M_{Z}\right) \lesssim 0.631 & \left(-0.50 \lesssim \log _{10} k\left(M_{Z}\right) \lesssim-0.20\right)\end{cases}
\end{aligned}
$$

$\log _{10}\left(m_{S} / 1 \mathrm{GeV}\right)$ and $\log _{10} k$ at the $M_{Z}$ scale, respectively. The boundaries of the plotted region are determined by the stability and triviality conditions. The words 'stability' and 'triviality' in both figures mean the corresponding conditions. We also show the contour satisfying $\Omega_{S} / \Omega_{\mathrm{DM}}=1$ with $\Omega_{\mathrm{DM}}=0.115$, where $\Omega_{S}$ and $\Omega_{\mathrm{DM}}$ are density parameters of the singlet DM and the observed value of the parameter [24], respectively. The contour is calculated by micrOMEGAs [25]. Since there is no DM candidate except for the $S$ to compensate for $\Omega_{S} / \Omega_{\mathrm{DM}}<1$, which is above the contour, we focus only on the contour. The relic density depends on $k$ and $m_{S}$ but not on $\lambda_{S}$, meanwhile $\lambda_{S}$ affects the stability and triviality bounds. In the figure, $\lambda_{S}\left(M_{Z}\right)$ is randomly varied from 0 to $4 \pi$, where the $\lambda_{S}$-dependence of the stability and triviality bounds is not stringent, and most of $\lambda_{S}\left(M_{Z}\right) \in[0,1]$ as the boundary condition can satisfy the bounds. A direct DM search experiment, XENON100 (2012), gives an exclu-
The future XENON100 experiment with 20 times higher sensitivity, which is described by the (blue) dotted lines in Fig. 2, will be able to completely rule out the lighter $m_{S}$ region $R_{1}$. On the other hand, the heavier $m_{S}$ region, $R_{2}$, can be currently allowed by all experiments searching for DM. It is seen that the future XENON100 $\times 20$ can check up to $m_{S} \lesssim 1,000 \mathrm{GeV}\left(\log _{10}\left(m_{S} / 1 \mathrm{GeV}\right) \lesssim 3\right)$. The future XENON1T experiment and combined data from indirect detections of Fermi+CTA+Planck at $1 \sigma$ CL may be able to reach up to $m_{S} \simeq 5 \mathrm{TeV}$ [12]. The lower and upper bounds on $m_{S}$ in the region $R_{1}$ come from the triviality bound on $\lambda$ and the XENON100 (2012) experiment, respectively. On the other hand, the lower and upper bounds on $m_{S}$ in the

\footnotetext{
7 The lighter DM mass region of $m_{S} \lesssim 62.5 \mathrm{GeV}\left(\log \left(m_{S} / 1 \mathrm{GeV}\right) \lesssim\right.$ 1.8) with $-1.8 \lesssim \log _{10} k$ is ruled out by the invisible Higgs decay into a pair of DM at the LHC [12]. But the stability and triviality cannot be realized in the region as shown in Fig. 2.
} 
region $R_{2}$ are given by the stability and triviality bounds on $\lambda$, respectively. Since $k$ in the R.H.S of Eq. (15) is effective only above the energy scale of $m_{S}$, the triviality bound on $\lambda$ becomes severe as the $m_{S}$ becomes small. We also find that the two regions $R_{1}$ and $R_{2}$ are almost the same as in the NNMSM [10]. This means that the differences in runnings of the gauge couplings do not affect the stability, triviality, and correct abundance of DM in this class of model. The favored region still depends on the top mass rather than the runnings of the gauge couplings as pointed out in Ref. [10]. In fact, the heavier mass region becomes narrow as the top mass becomes larger due to the stability bound, while the region $R_{1}$ does not depend on the top mass, because the triviality bound on $\lambda$ does not depend on the top Yukawa coupling (see Ref. [10] for detailed discussions of the top mass dependence of the region).

\subsection{Inflation, neutrinos, and baryogenesis}

In this section, realizations of inflation, suitable tiny active neutrino masses, and baryogenesis are discussed. The relevant Lagrangian for the inflaton is given by $\mathcal{L}_{\varphi}$ in Eq. (5). The WMAP [24,26] and the Planck [27] measurements of the cosmic microwave background (CMB) constrain the cosmological parameters related with the inflation in the early universe. In particular, the first results based on the Planck measurement with a WMAP polarization low-multipole likelihood at $\ell \leq 23$ (WP) [24,26] and high-resolution (highL) $\mathrm{CMB}$ data give

$n_{\mathrm{S}}=0.959 \pm 0.007(68 \% ;$ Planck $+\mathrm{WP}+$ highL $)$,

$r_{0.002}<\left\{\begin{array}{c}0.11(95 \% ; \text { no running, Planck }+\mathrm{WP}+\text { highL }) \\ 0.26(95 \% ; \text { including running, } \\ \text { Planck }+\mathrm{WP}+\text { highL })\end{array}\right.$,

$\mathrm{d} n_{\mathrm{S}} / \mathrm{d} \ln k=-0.015 \pm 0.017(95 \% ;$ Planck $+\mathrm{WP}+$ highL $)$

for the scalar spectrum power-law index, the ratio of tensor primordial power to curvature power, and the running of the spectral index, respectively, in the context of the $\triangle \mathrm{CDM}$ model. Regarding $r_{0.002}$, the constraints are given for the case of both no running and including running of the spectral indices.

We also adopt the same inflation model in NNMSM-II as in the NNMSM. The inflaton potential is the ColemanWeinberg (CW) type [28-32]. In this potential Eq. (5), the $\mathrm{VEV}$ of $\varphi$ becomes $\sigma$. When we take $(\phi, \sigma, B) \simeq$ $\left(6.60 \times 10^{19} \mathrm{GeV}, 9.57 \times 10^{19} \mathrm{GeV}, 10^{-15}\right)$, the model can lead to $n_{\mathrm{s}}=0.96, r=0.1, \mathrm{~d} n_{\mathrm{s}} / \mathrm{d} \ln k \simeq 8.19 \times 10^{-4}$, and $(\delta \rho / \rho) \sim \mathcal{O}\left(10^{-5}\right)$, which are consistent with the cosmological data. The values of the couplings of the infla- ton with the Higgs, DM, right-handed neutrinos, and new adjoint fermions are also constrained, because there is an upper bound on the reheating temperature after the inflation as $T_{\mathrm{RH}} \lesssim 1.86 \times 10^{8} \mathrm{GeV}$. This upper bound leads to $\mu_{1,2} \lesssim 6.86 \times 10^{7} \mathrm{GeV}$ and $\left(y_{N}^{i j}, y_{3}, y_{2}\right) \lesssim 1.79 \times 10^{-6}$. Since $\kappa_{H, S}$ should be almost vanishing at low energy for the realizations of the EW symmetry breaking and the DM mass, we take the values of $\kappa_{H, S}$ as very tiny at the epoch of inflation. The smallness of $\kappa_{H, S}$ also does not spoil the stability and triviality bounds. The lower bound of the reheating temperature depends on the baryogenesis mechanism. When the baryogenesis works through the sphaleron process, the reheating temperature must be at least higher than $\mathcal{O}\left(10^{2}\right) \mathrm{GeV}$.

The neutrino sector is shown in Eq. (4), where tiny active neutrino masses are obtained through the type-I and -III seesaw mechanisms. Since there are two right-handed neutrinos and one adjoint $\left(S U(2)_{L}\right.$ triplet) fermion, three active neutrinos are predicted to be massive in NNMSMII. The Yukawa coupling of the triplet fermion should be $\left|y_{v_{\alpha}}\right| \lesssim \mathcal{O}\left(10^{-6}\right)$ so as not to exceed the typical neutrino mass scale of $m_{v} \sim 0.1 \mathrm{eV}$. Thus, the region of $\mathcal{O}\left(10^{-13}\right) \lesssim\left|y_{v_{\alpha}}\right| \lesssim \mathcal{O}\left(10^{-6}\right)$ is allowed in which the lower bound comes from the discussion of the BBN as mentioned above. Recalling the reheating temperature in NNMSM-II, the masses of the right-handed neutrino must be lighter than $1.86 \times 10^{8} \mathrm{GeV}$. What mechanism can induce the suitable baryon asymmetry at such a low reheating temperature? One possibility is resonant leptogenesis [33] in which the righthanded neutrinos may be light, up to $1 \mathrm{TeV}$. Thus, the reheating temperature, $1 \mathrm{TeV} \lesssim T_{\mathrm{RH}} \lesssim 1.86 \times 10^{8} \mathrm{GeV}$, can realize resonant leptogenesis, which means for the couplings of the inflaton $369 \mathrm{GeV} \lesssim \mu_{1,2} \lesssim 6.86 \times 10^{7} \mathrm{GeV}$ and $9.63 \times 10^{-12} \lesssim\left(y_{N}^{i j}, y_{3}, y_{2}\right) \lesssim 1.79 \times 10^{-6}$ in Eq. (5).

\section{NNMSM type-III}

\subsection{Models}

We also discuss other possibilities, which are alternatives to the NNMSM and NNMSM-II, for realizing GCU by different particle contents. Here, we suggest a class of model with several generations of the adjoint fermions and without the right-handed neutrinos. We refer this class of models to NNMSM-III. We focus on two simple models, NNMSMIII-A and -B, in this class of models. Both NNMSM-III-A and $-B$ introduces three new fields such as one $\lambda_{3}$ and two generations of $\lambda_{2, i}(i=1,2)$ in addition to the singlet DM, inflaton, and the cosmological constant. Then, NNMSM-IIIA requires that the mass scales of the three new fields are the same and NNMSM-III-B allows different mass scales 
Table 2 Quantum numbers of additional particles in NNMSM-III-A and $-\mathrm{B}(i=1,2)$

\begin{tabular}{lllll}
\hline & $\lambda_{3}$ & $\lambda_{2, i}$ & $S$ & $\varphi$ \\
\hline$S U(3)_{C}$ & 8 & 1 & 1 & 1 \\
$S U(2)_{L}$ & 1 & 3 & 1 & 1 \\
$Z_{2}$ & + & + & - & + \\
\hline
\end{tabular}

between $\lambda_{3}$ and $\lambda_{2, i}$. The quantum numbers of these particles for both models are given in Table 2 .

Only the singlet scalar DM has odd parity under an additional $Z_{2}$ symmetry like NNMSM-II. Since the runnings of the GCU are still changed from the previous models, we will focus on them later.

NNMSM-III-A and -B are also presented as renormalizable theory. The most general form of the Lagrangian allowed by the symmetries and renormalizability is given by

$$
\begin{aligned}
\mathcal{L}_{\mathrm{NNMSM}}=\mathcal{L}_{\mathrm{SM}}+\mathcal{L}_{S}+\mathcal{L}_{\varphi}+\mathcal{L}_{\Lambda}+\mathcal{L}^{\prime} \\
\mathcal{L}_{\varphi}=-B \varphi^{4}\left[\ln \left(\frac{\varphi^{2}}{\sigma^{2}}\right)-\frac{1}{2}\right]-\frac{B \sigma^{4}}{2}-\mu_{1} \varphi|H|^{2} \\
\quad-\mu_{2} \varphi S^{2}-\kappa_{H} \varphi^{2}|H|^{2}-\kappa_{S} \varphi^{2} S^{2} \\
\quad-\left(y_{3} \varphi \bar{\lambda}_{3} \lambda_{3}+y_{2}^{i j} \varphi \overline{\lambda_{2 i}} \lambda_{2 j}+\text { c.c. }\right)+(\text { kinetic term }),
\end{aligned}
$$

$$
\begin{aligned}
\mathcal{L}^{\prime}= & -\left(y_{\nu_{\alpha}}^{i} \overline{L_{\alpha}} \lambda_{2, i} \tilde{H}+M_{3} \overline{\lambda_{3}} \lambda_{3}+M_{2 i} \overline{\lambda_{2 i}} \lambda_{2 i}+\text { h.c. }\right) \\
& + \text { (kinetic terms) }
\end{aligned}
$$

with

$M_{\mathrm{NP}} \equiv M_{3}=M_{2, i}$ for NNMSM-III-A

$M_{3} \neq M_{2, i}, \quad M_{2,1}=M_{2,2} \quad$ for NNMSM-III-B'

where the inflaton potential in $\mathcal{L}_{\varphi}$ is the same as those of the other NNMSMs, but the interactions are different from them. Then, $\mathcal{L}^{\prime}$ is a new Lagrangian for the models. The mass matrix $M_{2}$ is assumed to be diagonal for simplicity. The Lagrangians $\mathcal{L}_{\mathrm{SM}}+\mathcal{L}_{S}+\mathcal{L}_{\Lambda}$ are the same as those of the NNMSMs. ${ }^{8}$ One of the advantages of NNMSM-III-A is that we do not need two right-handed neutrinos and different mass scales between $\lambda_{3}$ and $\lambda_{2, i}$ for the realization of the GCU, unlike NNMSM-II as we will show later. Thus, we introduce one mass scale for the new adjoint fermions and define it as $M_{\mathrm{NP}} \equiv M_{3}=M_{2, i}$ for NNMSM-III-A. NNMSM-III-B is a generalization of NNMSM-III-A.

\subsection{Gauge coupling unification}

First, we investigate the runnings of the gauge couplings in the NNMSM-III-A and B. Since we introduce three adjoint

\footnotetext{
8 We also simply assume that the origin of DE is the tiny cosmological constant as in the other NNMSMs.
}

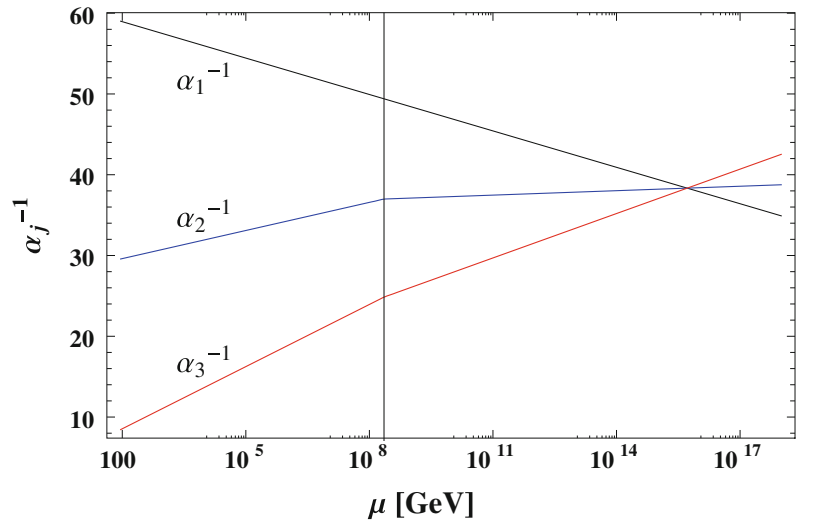

Fig. 3 The runnings of the gauge couplings in the NNMSM-III-A. The meanings of the figure are the same as in Fig. 1 . We take $M_{\mathrm{NP}}=$ $2.26 \times 10^{8} \mathrm{GeV}$, and the coupling unification is realized at $\mu=\Lambda_{\mathrm{GUT}} \simeq$ $5.20 \times 10^{15} \mathrm{GeV}$ with $\alpha_{\mathrm{GCU}}^{-1} \simeq 38.3$

fermions $\lambda_{3}$ and $\lambda_{2, i}(i=1,2)$, as listed in Table 2, the beta functions for the gauge couplings are modified to

$$
\begin{aligned}
\left(b_{1}^{\mathrm{SM}}, b_{2}^{\mathrm{SM}}, b_{3}^{\mathrm{SM}}\right) & =\left(\frac{41}{10},-\frac{19}{6},-7\right), \\
\left(b_{1}^{\lambda_{3}}, b_{2}^{\lambda_{3}}, b_{3}^{\lambda_{3}}\right) & =(0,0,2), \\
\left(b_{1}^{\lambda_{2}}, b_{2}^{\lambda_{2}}, b_{3}^{\lambda_{2}}\right) & =\left(0, \frac{8}{3}, 0\right) .
\end{aligned}
$$

\subsubsection{NNMSM-III-A case}

According to the numerical analyses, taking the free parameter $M_{\mathrm{NP}}$ as $2.26 \times 10^{8} \mathrm{GeV}$ in NNMSM-III-A can realize GCU with a good precision at 1-loop level, as shown in Fig. $3 .{ }^{9}$

Since all masses of the new adjoint fermions lie around the same scale, $M_{Z}<M_{\mathrm{NP}}=M_{3}=M_{2}$, we should utilize the RGEs of Eq. (8) with $b_{j}^{\mathrm{SM}}+b_{j}^{\lambda_{3}}+b_{j}^{\lambda_{2}}$ given in Eq. (29) at the high-energy scale $\left(M_{\mathrm{NP}} \leq \mu\right)$, while the right-handed side of Eq. (8) must be $b_{j}^{\mathrm{SM}}$ at the low-energy scale $\left(M_{Z} \leq\right.$ $\mu<M_{\mathrm{NP}}$ ). We show the threshold of new particles with $2.26 \times 10^{8} \mathrm{GeV}$ mass by a black solid line. The NNMSMIII-A suggests GCU at

$\Lambda_{\mathrm{GCU}} \simeq 5.20 \times 10^{15} \mathrm{GeV}$

with the unified coupling as

$\alpha_{\mathrm{GCU}}^{-1} \simeq 38.3$.

The model also predicts the proton lifetime as $\tau=$ $1.94_{-0.80}^{+2.06} \times 10^{35}$ years for $\alpha_{\mathrm{H}}=-0.0112 \pm 0.0034 \mathrm{GeV}^{3}$. Therefore, this model can satisfy the constraint on the proton decay even with the most conservative value of $\alpha_{\mathrm{H}}$, but it

\footnotetext{
${ }^{9}$ We take the same values of the parameters in EW theory as in the previous model.
} 


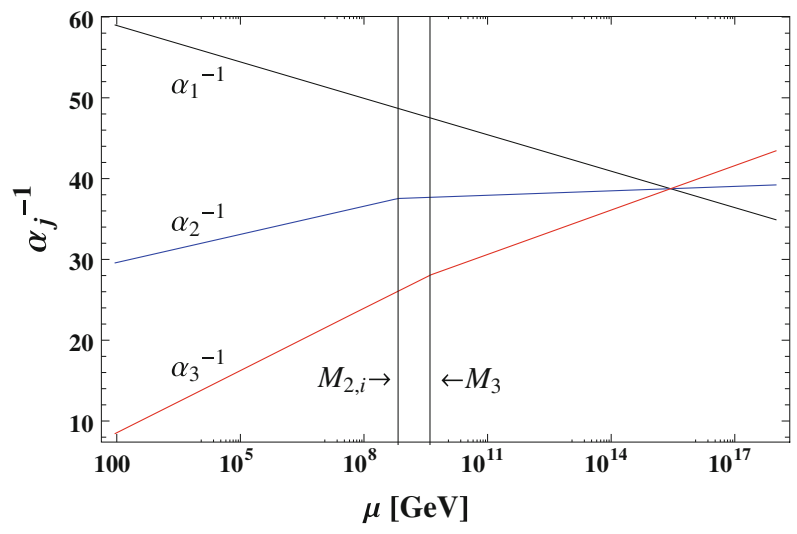

Fig. 4 The runnings of the gauge couplings in the NNMSM-III-B. The meanings of the figure are the same as in Figs. 1 and 3. We take $M_{3} \simeq$ $4 \times 10^{9} \mathrm{GeV}$ and $M_{2, i} \simeq 6.73 \times 10^{8} \mathrm{GeV}$, and the coupling unification is realized at $\mu=\Lambda_{\mathrm{GUT}} \simeq 2.77 \times 10^{15} \mathrm{GeV}$ with $\alpha_{\mathrm{GCU}}^{-1} \simeq 38.8$

might be difficult to check the proton decay in this model by the future Hyper-Kamiokande experiment.

There are other initial setups for realizing GCU with the same mass scales for the new adjoint fermions, i.e., more generations of adjoint fermions can also lead to GCU. Some examples are given in the Appendix.

\subsubsection{NNMSM-III-B case}

Since one can generally take different mass scales between $M_{3}$ and $M_{2, i}$, we consider the simplest case in the generalization, which is NNMSM-III-B. According to the numerical analyses, when we take $M_{3} \simeq 4 \times 10^{9} \mathrm{GeV}$ and $M_{2, i} \simeq 6.73 \times 10^{8} \mathrm{GeV}$, GCU can be realized as shown in Fig. 4.

The beta functions in NNMSM-II are

$b_{j}= \begin{cases}b_{j}^{\mathrm{SM}} & \text { for } M_{Z} \leq \mu<M_{2, i} \\ b_{j}^{\mathrm{SM}}+b_{j}^{\lambda_{2}} & \text { for } M_{2, i} \leq \mu<M_{3}, \\ b_{j}^{\mathrm{SM}}+b_{j}^{\lambda_{2}}+b_{j}^{\lambda_{3}} & \text { for } M_{3} \leq \mu\end{cases}$

with Eq. (29). We show the thresholds of the new particles with masses $M_{3} \simeq 4 \times 10^{9} \mathrm{GeV}$ and $M_{2, i} \simeq 6.73 \times 10^{8} \mathrm{GeV}$ by black solid lines. This case suggests the GCU to occur at

$\Lambda_{\mathrm{GCU}} \simeq 2.77 \times 10^{15} \mathrm{GeV}$

with the unified coupling as

$\alpha_{\mathrm{GCU}}^{-1} \simeq 38.8$.

This mass spectrum interestingly predicts the proton lifetime as $\tau=1.50_{-0.62}^{+1.60} \times 10^{34}$ years for $\alpha_{\mathrm{H}}=-0.0112 \pm$ $0.0034 \mathrm{GeV}^{3}$. Therefore, the model with this mass spectrum can satisfy the constraint on the proton decay even with the most conservative value of $\alpha_{\mathrm{H}}$ and it can be checked by the future Hyper-Kamiokande experiment if the model is correct.
This is one advantage compared to NNMSM-III-A. In fact, there is an upper bound on the mass scale of $M_{3}$ (or $M_{2, i}$ ), which just comes from the proton decay. Therefore, in a broad region of $M_{3} \lesssim 4 \times 10^{9} \mathrm{GeV}$ (or $M_{2,1} \lesssim 6.73 \times 10^{8} \mathrm{GeV}$ ), there are solutions for the realization of GCU. There are also initial setups for the GCU with different mass scales between $\lambda_{3}$ and $\lambda_{2, i}$ and more generations of them. Some simple examples are given in the Appendix.

\subsection{Abundance and stability of new fermions}

We also adopt the few production scenario for the colored particle $\lambda_{3}$ in NNMSM-III to avoid the problem of the presence of the colored particle. The reheating temperature should be

$T_{\mathrm{RH}}<\left\{\begin{array}{ll}5.65 \times 10^{6} \mathrm{GeV} & \text { for NNMSM-III-A } \\ 10^{8} \mathrm{GeV} & \text { for NNMSM-III-B }\end{array}\right.$.

Note that $\lambda_{2, i}$ are not also thermally produced because of $M_{\lambda_{2, i}}>T_{\mathrm{RH}}$ in the models.

\subsection{Stability, triviality, and dark matter}

Regarding the stability, triviality, and DM, since the differences in the runnings of the gauge couplings almost do not affect the stability and triviality bounds, the favored regions in NNMSM-III-A and -B are also almost the same as in NNMSM-II. Thus, NNMSM-III-A and -B predict the same mass region of DM and $k$ as in the other NNMSMs.

\subsection{Inflation, neutrinos, and baryogenesis}

Realizations of inflation, a suitable tiny active neutrino mass and baryogenesis in NNMSM-III are discussed in this section. Regarding the inflation model, the same CW type inflaton potential as in the previous models is utilized in the models, but the upper bounds on the inflaton couplings are changed due to different constraints on the reheating temperature. The upper bounds on the inflaton couplings are

$\mu_{1,2} \lesssim \begin{cases}2.09 \times 10^{6} \mathrm{GeV} & \text { for NNMSM-III-A } \\ 3.69 \times 10^{7} \mathrm{GeV} & \text { for NNMSM-III-B }\end{cases}$

$y_{3}, y_{2} \lesssim\left\{\begin{array}{ll}5.44 \times 10^{-8} & \text { for NNMSM-III-A } \\ 9.63 \times 10^{-7} & \text { for NNMSM-III-B }\end{array}\right.$.

Since NNMSM-III does not include the right-handed neutrinos, the neutrino sector is changed from NNMSM-II. In NNMSM-III, the tiny active neutrino mass can be realized by $S U(2)_{L}$ adjoint fermions through the type-III seesaw mechanism. The relevant Lagrangian is given by Eq. (27). Since both NNMSM-III-A and -B have only two generations of $\lambda_{2, i}$, one of the active neutrinos is predicted to be massless, $m_{1}=0\left(m_{3}=0\right)$ for the normal (inverted) mass hierar- 
chy. Recalling the condition $M_{\lambda_{2, i}}>T_{\mathrm{RH}}$ in NNMSM-III-A and -B for the few production scenario, $\lambda_{2, i}$ do not play a role for generating the baryon asymmetry of the universe. What mechanism can induce the BAU? One possibility is baryogenesis from the dark sector [34,35], which presupposes an asymmetry between DM and anti-DM in which the asymmetry of the dark matter sector including a new dark matter number can be converted into the lepton number. ${ }^{10}$ As a result, the baryon number can be generated through the sphaleron process. This means that the reheating temperature should be typically $\mathcal{O}\left(10^{2}\right) \mathrm{GeV} \lesssim T_{\mathrm{RH}}$ for both NNMSM-III-A and -B, which leads to $\mathcal{O}(10) \mathrm{GeV} \lesssim \mu_{1,2}$ and $\mathcal{O}\left(10^{-13}\right) \lesssim y_{3}, y_{2}$.

\section{Summary}

There are some unsolved problems in the SM. These are, for instance, explanations for DM, the gauge hierarchy problem, tiny neutrino mass scales, baryogenesis, inflation, and DE. The extended SM without the SUSY, the so-called NNMSM, could explain the above problems except for the gauge problem by adding two adjoint fermions, four vector-like fermions, two gauge singlet real scalars, two right-handed neutrinos, and a small cosmological constant. In this paper, we suggested two types of alternatives (NNMSM-II and NNMSM-III) to the NNMSM by reducing additional fields while keeping the above merits of the NNMSM.

First, we have taken a setup where the new fermions have a different mass scale of new physics. Under this condition, GCU with the proton stability determines the field contents of NNMSM-II, i.e., two adjoint fermions are added to the SM in addition to two gauge singlet real scalars, two righthanded neutrinos, and a small cosmological constant. The GCU can occur at $\Lambda_{\mathrm{GCU}} \simeq 2.41 \times 10^{15} \mathrm{GeV}$ with two mass scales of new particles as $M_{3} \simeq 7.44 \times 10^{9} \mathrm{GeV}$ and $M_{2}=300 \mathrm{GeV}$. We consider the reheating temperature as $T_{\mathrm{RH}} \lesssim 1.86 \times 10^{8} \mathrm{GeV}$ in order not to produce stable adjoint fermions in the early universe. This reheating temperature requires the following issues. The masses of the right-handed neutrino should be smaller than $1.86 \times 10^{8} \mathrm{GeV}$, so that a tiny neutrino mass is realized through the type-I seesaw mechanism with relatively small neutrino Yukawa couplings. The BAU should be achieved, for example, through resonant leptogenesis. We have also analyzed the stability and triviality conditions by use of recent experimental data of the Higgs and top masses. We found the parameter regions in which the correct abundance of DM can also be realized at the same time. One is the lighter $m_{S}$ region: $63.5 \mathrm{GeV} \lesssim m_{S} \lesssim 64.0 \mathrm{GeV}$, and the other is the heavier

\footnotetext{
10 This mechanism can be employed in the other NNMSMs if one supposes the above asymmetry to hold for the DM sector in the models.
}

region: $708 \mathrm{GeV} \lesssim m_{S} \lesssim 2040 \mathrm{GeV}$ with the center value of top pole mass. Both regions are almost the same as in the NNMSM. This means that the differences in runnings of the gauge couplings between NNMSM and NNMSM-II do not affect the stability, triviality, and correct abundance of DM in these classes of model. Therefore, the favored region for the stability and triviality still depends on the top mass rather than the running of the gauge couplings. The future XENON100 experiment with 20 times higher sensitivity will completely check out the lighter mass region. On the other hand, the heavier mass region will also be completely checked by the future direct experiments of XENON $100 \times 20$, XENON1T and/or combined data from indirect detections of Fermi + CTA+Planck at $1 \sigma \mathrm{CL}$.

Second, we have also taken a different setup (NNMSMIII), which includes three adjoint fermions $\left(\lambda_{3}\right.$ and $\lambda_{2, i}$ $(i=1,2))$ in addition to two gauge singlet real scalars and a small cosmological constant, but it does not have righthanded neutrinos. Removing the right-handed neutrino is one of the advantages of this setup. Then, we have considered two simple cases in this setup. One is that all masses of adjoint fermions are the same. The other is that masses of the $S U(3)_{C}$ and $S U(2)_{L}$ adjoint fermions are different. The first and the second cases are named NNMSM-III-A and -B, respectively. The GCU can occur at $\Lambda_{\mathrm{GCU}} \simeq 5.20(2.77) \times 10^{15} \mathrm{GeV}$ with $M_{3}=M_{2, i} \simeq 2.26 \times 10^{8}\left(M_{3} \simeq 4 \times 10^{9}\right.$ and $M_{2, i} \simeq$ $\left.6.73 \times 10^{8}\right) \mathrm{GeV}$ in NNMSM-III-A (-B). Thus, the reheating temperature should be $T_{\mathrm{RH}} \lesssim 5.65 \times 10^{6}\left(10^{8}\right) \mathrm{GeV}$ for model A (B). The tiny neutrino mass can be realized by two adjoint fermions under $S U(2)_{L}$ through the type-III seesaw mechanism, and the BAU can be achieved, e.g., baryogenesis from the dark sector in both models. We have also investigated other initial setups for realizing GCU in the appendix. These have several generations of $\lambda_{3}$ and/or $\lambda_{2}$. Regarding the stability, triviality, and DM in NNMSM-III, since the differences in the runnings of the gauge couplings almost do not affect the stability and triviality bounds, the favored regions in NNMSM-III are almost the same as in the other NNMSMs. Therefore, NNMSM-III predicts the same mass region of DM and $k$ as the previous models.

Finally, we briefly compare the NNMSMs to any other minimal extensions of the SM such as the minimal leftright model (e.g., see [7]), neutrino minimal standard model ( $v$ MSM) [8,9], and some supersymmetric extensions. In the context of the left-right model, GCU, DM, tiny neutrino mass, and BAU can be explained, but there is no complete analysis for the vacuum stability and triviality with the latest center values of the Higgs and top mass. Such a discussion might be interesting, although the RGEs for the Higgs sector are more complicated than that in the NNMSMs. The $\nu$ MSM with three right-handed neutrinos is one simple extension of the SM, and can explain some problems (DM, tiny neutrino mass, and BAU) at the same time. To achieve GCU in the 
vMSM, some additional particles are still needed. In addition, the Higgs sector of the vMSM is the same as in the SM, and thus the vacuum in the model becomes unstable before the Planck scale. The degrees of freedom in the NNMSMs are fewer than general extensions of the SM with the left-right symmetry and supersymmetry. These are the advantages of the NNMSMs.

Acknowledgments We are grateful to Shigeki Matsumoto and Osamu Seto for useful discussions. This work is partially supported by Scientific Grant by Ministry of Education and Science, Nos. 00293803, 20244028, 21244036, 23340070, and by the SUHARA Memorial Foundation. The works of K.K. and R.T. are supported by Research Fellow- ships of the Japan Society for the Promotion of Science for Young Scientists.

Open Access This article is distributed under the terms of the Creative Commons Attribution License which permits any use, distribution, and reproduction in any medium, provided the original author(s) and the source are credited.

Funded by $\mathrm{SCOAP}^{3}$ / License Version CC BY 4.0.

\section{Appendix A: Other initial setups for realizing GCU}

Let us investigate other initial setups for realizing GCU in this appendix.
Table 3 Examples for realizing GCU with several generations of new adjoint fermions under the condition of $M_{\mathrm{NP}}=M_{3, i}=$ $M_{2, i}$ in NNMSM-III
The label ' $*$ ' on the number of $N_{\lambda_{2}}$ means that the cases cannot satisfy the proton decay constraint. The label ' $\dagger$ ' means that the cases never realize GCU. The label ' $\ddagger$ ' means that the cases can realize GCU but the scale is higher than the Planck scale. The cases without any labels can realize GCU at an energy scale lower than the Planck scale and satisfy the proton decay constraint

\begin{tabular}{|c|c|c|c|c|c|}
\hline$N_{\lambda_{3}}$ & $N_{\lambda_{2}}$ & $M_{\mathrm{NP}}(\mathrm{GeV})$ & $\Lambda_{\mathrm{GCU}}\left(10^{15} \mathrm{GeV}\right)$ & $\alpha_{\mathrm{GCU}}^{-1}$ & $\tau\left(10^{33}\right.$ years $)$ \\
\hline 1 & $3^{*}$ & $2.21 \times 10^{11}$ & 1.41 & 39.3 & $<8.2 \times 10^{33}$ years \\
\hline \multirow[t]{4}{*}{2} & $1,2^{\dagger}$ & - & - & - & - \\
\hline & 3 & $5.80 \times 10^{9}$ & 99.2 & 36.4 & $2.88_{-1.19}^{+3.06} \times 10^{7}$ \\
\hline & 4 & $1.09 \times 10^{12}$ & 5.20 & 38.3 & $1.89_{-0.78}^{+2.00} \times 10^{2}$ \\
\hline & $\geq 5^{*}$ & $\geq 6.47 \times 10^{12}$ & $\leq 1.90$ & $\geq 39.0$ & $<8.2 \times 10^{33}$ years \\
\hline \multirow[t]{5}{*}{3} & $1-3^{\dagger}$ & - & - & - & - \\
\hline & $4^{\ddagger}$ & $1.33 \times 10^{11}$ & $>M_{\mathrm{pl}}$ & 34.6 & $>\mathcal{O}\left(10^{35}\right)$ years \\
\hline & 5,6 & $(0.52-1.83) \times 10^{13}$ & $(5.20-23.0)$ & $(37.3-38.3)$ & $>\mathcal{O}\left(10^{35}\right)$ years \\
\hline & 7 & $3.47 \times 10^{13}$ & 2.45 & 38.8 & $8.94_{-3.68}^{+9.49}$ \\
\hline & $\geq 8^{*}$ & $\geq 5.11 \times 10^{13}$ & $\leq 1.55$ & $\geq 39.1$ & $<8.2 \times 10^{33}$ years \\
\hline \multirow[t]{5}{*}{4} & $1-4^{*}$ & - & - & - & - \\
\hline & $5^{\ddagger}$ & $2.74 \times 10^{12}$ & $>M_{\mathrm{pl}}$ & 32.8 & $>\mathcal{O}\left(10^{35}\right)$ years \\
\hline & $6-8$ & $(2.39-7.51) \times 10^{13}$ & $(5.20-99.2)$ & $(36.4-38.3)$ & $>\mathcal{O}\left(10^{35}\right)$ years \\
\hline & 9 & $9.49 \times 10^{13}$ & 2.85 & 38.7 & $16.4_{-6.75}^{+17.4}$ \\
\hline & $\geq 10^{\dagger}$ & $\geq 1.11 \times 10^{14}$ & $\leq 1.90$ & $\geq 39.0$ & $<8.2 \times 10^{33}$ years \\
\hline \multirow[t]{6}{*}{5} & $1-5^{*}$ & - & - & - & - \\
\hline & $6^{\ddagger}$ & $5.11 \times 10^{12}$ & $>M_{\mathrm{pl}}$ & 31.0 & $>\mathcal{O}\left(10^{35}\right)$ years \\
\hline & $7-10$ & $(1.75-7.51) \times 10^{14}$ & $(5.20-417)$ & $(35.5-38.3)$ & $>\mathcal{O}\left(10^{35}\right)$ years \\
\hline & 11 & $1.85 \times 10^{14}$ & 3.15 & 38.7 & $24.6_{-10.1}^{+26.1}$ \\
\hline & 12 & $1.93 \times 10^{14}$ & 2.20 & 38.9 & $5.76_{-2.37}^{+6.61}$ \\
\hline & $\geq 13^{\dagger}$ & $\geq 1.99 \times 10^{14}$ & $\leq 1.68$ & $\geq 39.1$ & $<8.2 \times 10^{33}$ years \\
\hline \multirow[t]{6}{*}{6} & $1-6^{*}$ & - & - & - & - \\
\hline & $7,8^{\ddagger}$ & $(4.76-8.67) \times 10^{14}$ & $>M_{\mathrm{pl}}$ & $(29.3-34.6)$ & $>\mathcal{O}\left(10^{35}\right)$ years \\
\hline & $9-12$ & $(3.09-3.85) \times 10^{14}$ & $(5.20-99.2)$ & $(36.4-38.3)$ & $>\mathcal{O}\left(10^{35}\right)$ years \\
\hline & 13 & $2.99 \times 10^{14}$ & 3.39 & 38.6 & $32.8_{-13.5}^{+34.9}$ \\
\hline & 14 & $2.92 \times 10^{14}$ & 2.45 & 38.8 & $8.90_{-3.66}^{+9.45}$ \\
\hline & $\geq 15^{\dagger}$ & $\geq 2.86 \times 10^{14}$ & $\leq 1.90$ & $\geq 39.0$ & $<8.2 \times 10^{33}$ years \\
\hline \multirow[t]{7}{*}{7} & $1-7^{*}$ & - & - & - & - \\
\hline & $8,9^{\ddagger}$ & $(0.204-1.34) \times 10^{16}$ & $>M_{\mathrm{pl}}$ & $(27.7-33.7)$ & $>\mathcal{O}\left(10^{35}\right)$ years \\
\hline & $10-14$ & $(0.462-1.04) \times 10^{15}$ & $(5.20-259)$ & $(35.8-38.3)$ & $>\mathcal{O}\left(10^{35}\right)$ years \\
\hline & 15 & $4.27 \times 10^{14}$ & 3.57 & 38.6 & $40.8_{-16.8}^{+43.3}$ \\
\hline & 16 & $4.02 \times 10^{14}$ & 2.66 & 38.8 & $12.5_{-5.14}^{+13.3}$ \\
\hline & 17 & $3.83 \times 10^{14}$ & 2.10 & 38.9 & $4.83_{-1.99}^{+5.13}$ \\
\hline & $\geq 18^{\dagger}$ & $\leq 3.68 \times 10^{14}$ & $\leq 1.74$ & $\geq 39.1$ & $<8.2 \times 10^{33}$ years \\
\hline
\end{tabular}


Table 4 Examples for realizing GCU with several generations of new adjoint fermions and the condition of $M_{3, i} \neq M_{2, i}$ in NNMSM-III
All cases listed in this table can satisfy the proton decay constraint, but the cases with ' $\star$ ' are excluded by the LHC experiment searching for the $S U(2)_{L}$ adjoint fermion

\section{A.1 $M_{3, i}=M_{2, i}$ case}

Some examples of other initial setups for GCU with the condition of $M_{3, i}=M_{2, i}$ are given in Table 3. In the table, $N_{\lambda_{3}}$ and $N_{\lambda_{2}}$ are the number of generations of $\lambda_{3}$ and $\lambda_{2}$, respectively. We make some comments on those initial setups:

- The case of $\left(N_{\lambda_{3}}, N_{\lambda_{2}}\right)=(1,3)$ can also realize GCU but the case cannot satisfy the constraint from the proton decay. Such a case is labeled by ' $*$ ' on the number of $N_{\lambda_{2}}$.

- The cases of larger number of $N_{\lambda_{3}}$ need a larger number of $N_{\lambda_{2}}$ for the realization of GCU. For instance, GCU can occur from $\left(N_{\lambda_{3}}, N_{\lambda_{2}}\right)=(2, \geq 3)$. The cases that never realize GCU, e.g., $\left(N_{\lambda_{3}}, N_{\lambda_{2}}\right)=(2,1)$, are labeled by ' $\dagger$ '.

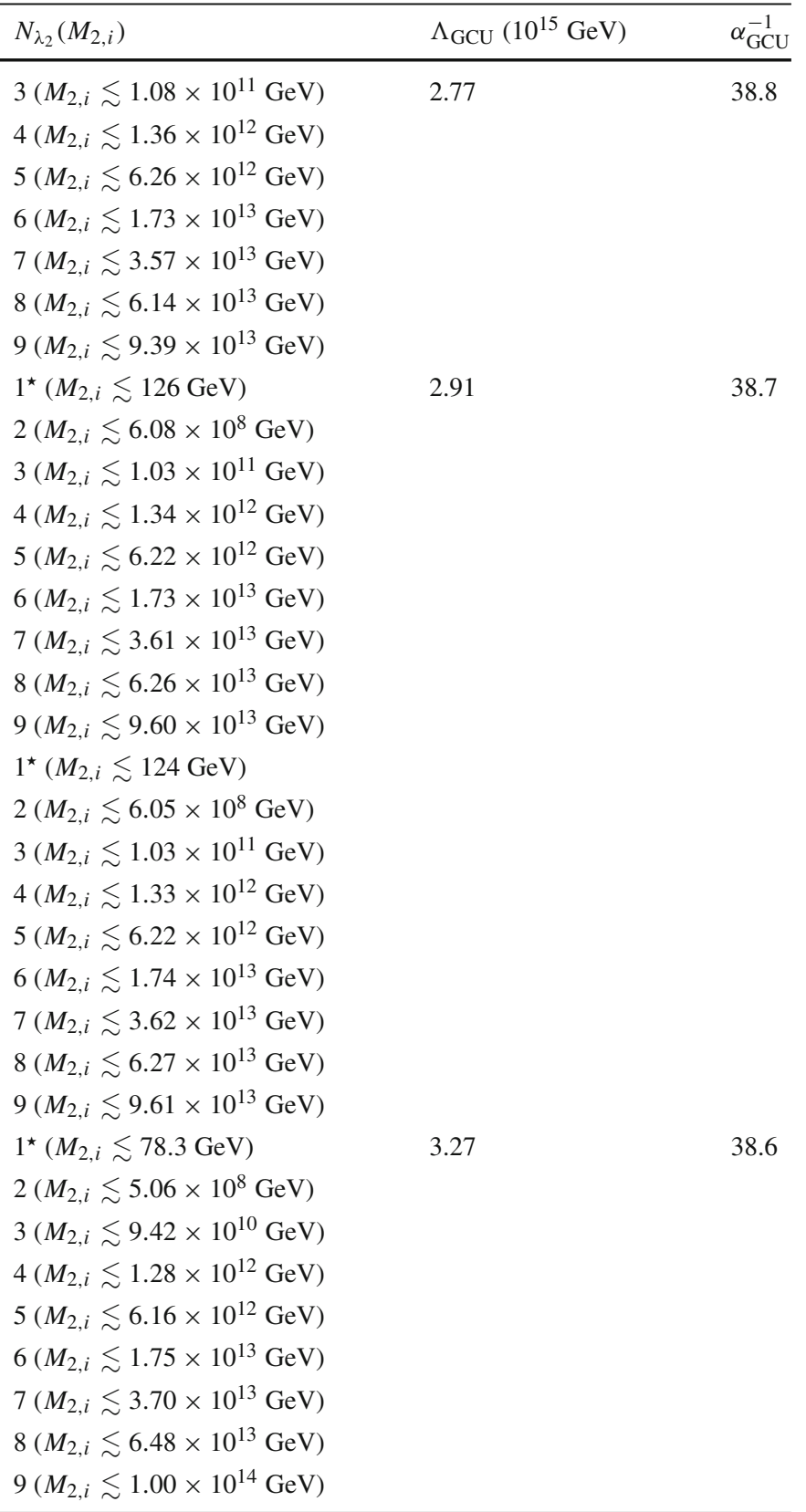

- There are upper bounds on the number of $N_{\lambda_{2}}$, which come from the constraint of the proton decay, for each case of $N_{\lambda_{3}}$. The cases exceeding the corresponding upper bound are also labeled by ' $*$ ', e.g., $\left(N_{\lambda_{3}}, N_{\lambda_{2}}\right)=(2, \geq 5)$ case.

- There are some combinations such that GCU can be realized but the GCU scale exceeds the Planck scale, e.g., $\left(N_{\lambda_{3}}, N_{\lambda_{2}}\right)=(3,4)$ and $(4,5)$, etc. The cases are labeled by ' $\ddagger$ '.

A. $2 M_{3, i} \neq M_{2, i}$ case

Some examples of other initial setups for the GCU allowing $M_{3, i} \neq M_{2, i}$ are given in Table 4. We make some comments on those initial setups: 
- Upper bounds on $M_{3, i}$ and $M_{2, i}$ are given for each case. We take a conservative limit of $\tau \gtrsim 10^{34}$ years for the proton decay to obtain the upper bounds. Therefore, all cases listed in Table 4 can satisfy the proton decay constraint.

- $\left(N_{\lambda_{3}}, N_{\lambda_{2}}\right)=(1,1)$ case is just NNMSM-II.

- $\left(N_{\lambda_{3}}, N_{\lambda_{2}}\right)=(1,2)$ case with $M_{3}=M_{2, i}\left(M_{3} \neq M_{2, i}\right)$ is NNMSM-III-A (-B).

- Larger number of $N_{\lambda_{3}}$ leads to larger upper bound on $M_{\lambda_{3, i}}$.

- The cases with $N_{\lambda_{2, i}}=1$ can satisfy the constraint from the proton decay but these are excluded by the LHC experiment searching for the $S U(2)_{L}$ triplet particle. The cases are labeled by ' $\star$ '.

Hierarchical mass spectra for $M_{3, i}$ and $M_{2, i}$ such as $M_{3,1}<$ $M_{3,2}$ might also realize GCU but we do not consider the hierarchical mass spectra case because of the minimality of the models in this work.

\section{References}

1. G. Aad et al., ATLAS Collaboration, Phys. Lett. B 716, 1 (2012) [arXiv:1207.7214 [hep-ex]]

2. S. Chatrchyan et al., CMS Collaboration, JHEP 1306, 081 (2013) [arXiv:1303.4571 [hep-ex]]

3. H. Davoudiasl, R. Kitano, T. Li, H. Murayama, Phys. Lett. B 609, 117 (2005) [arXiv:hep-ph/0405097]

4. M. Ibe, JHEP 0908, 086 (2009) [arXiv:0906.4667 [hep-ph]]

5. S. Baek, P. Ko, W.-I. Park, JHEP 1307, 013 (2013) [arXiv:1303. 4280 [hep-ph]]

6. S. Choi, S. Jung, P. Ko, JHEP 1310, 225 (2013) [arXiv:1307.3948 [hep-ph]]

7. R.E. Marshak, R.N. Mohapatra, Phys. Lett. B 91, 222 (1980)

8. T. Asaka, S. Blanchet, M. Shaposhnikov, Phys. Lett. B 631, 151 (2005) [arXiv:hep-ph/0503065]

9. T. Asaka, M. Shaposhnikov, Phys. Lett. B 620, 17 (2005) [arXiv: hep-ph/0505013]

10. N. Haba, K. Kaneta, R. Takahashi, [arXiv:1309.1231 [hep-ph]]

11. J. Beringer et al., Particle Data Group Collaboration, Phys. Rev. D 86, 010001 (2012)
12. J. M. Cline, K. Kainulainen, P. Scott, C. Weniger, Phys. Rev. D 88, 055025 (2013) [arXiv:1306.4710 [hep-ph]]

13. J. Hisano, [arXiv:hep-ph/0004266]

14. K.S. Babu, R.N. Mohapatra, Phys. Lett. B 715, 328 (2012) [arXiv: 1206.5701 [hep-ph]]

15. Y. Aoki et al., RBC-UKQCD Collaboration, Phys. Rev. D 78, 054505 (2008) [arXiv:0806.1031 [hep-lat]]

16. K. Abe, T. Abe, H. Aihara, Y. Fukuda, Y. Hayato, K. Huang, A.K. Ichikawa, M. Ikeda et al., [arXiv:1109.3262 [hep-ex]]

17. S. Chatrchyan et al., CMS Collaboration, Phys. Lett. B 718, 348 (2012) [arXiv:1210.1797 [hep-ex]]

18. The ATLAS Collaboration, ATLAS-CONF-2013-019

19. F. del Aguila, J.A. Aguilar-Saavedra, Nucl. Phys. B 813, 22 (2009) [arXiv:0808.2468 [hep-ph]]

20. G.F. Giudice, A. Romanino, Nucl. Phys. B 699, 65 (2004) [Erratum-ibid. B 706 (2005) 65] [arXiv:hep-ph/0406088]

21. P.F. Smith, J.R.J. Bennett, G.J. Homer, J.D. Lewin, H.E. Walford, W.A. Smith, Nucl. Phys. B 206, 333 (1982)

22. T.K. Hemmick, D. Elmore, T. Gentile, P.W. Kubik, S.L. Olsen, D. Ciampa, D. Nitz, H. Kagan et al., Phys. Rev. D 41, 2074 (1990)

23. H. Baer, K.-m. Cheung, J.F. Gunion, Phys. Rev. D 59, 075002 (1999) [arXiv:hep-ph/9806361]

24. C. L. Bennett et al., WMAP Collaboration Astrophys. J. Suppl. 208, 20 (2013) [arXiv:1212.5225 [astro-ph.CO]]

25. G. Belanger, F. Boudjema, A. Pukhov, A. Semenov [arXiv:1305. 0237 [hep-ph]]

26. G. Hinshaw et al., WMAP Collaboration [arXiv:1212.5226 [astroph.CO]]

27. P.A.R. Ade et al., Planck Collaboration [arXiv:1303.5076 [astroph.CO]]

28. S.R. Coleman, E.J. Weinberg, Phys. Rev. D 7, 1888 (1973)

29. Q. Shafi, A. Vilenkin, Phys. Rev. Lett. 52, 691 (1984)

30. S.-Y. Pi, Phys. Rev. Lett. 52, 1725 (1984)

31. L. Knox, M.S. Turner, Phys. Rev. Lett. 70, 371 (1993) [arXiv: astro-ph/9209006]

32. Q. Shafi, V.N. Senoguz, Phys. Rev. D 73, 127301 (2006) [arXiv: astro-ph/0603830]

33. A. Pilaftsis, T.E.J. Underwood, Nucl. Phys. B 692, 303 (2004) [arXiv:hep-ph/0309342]

34. J. Shelton, K.M. Zurek, Phys. Rev. D 82, 123512 (2010) [arXiv: 1008.1997 [hep-ph]]

35. N. Haba, S. Matsumoto, Prog. Theor. Phys. 125, 1311 (2011) [arXiv:1008.2487 [hep-ph]] 